Claremont Colleges

Scholarship@ Claremont

All HMC Faculty Publications and Research

HMC Faculty Scholarship

$10-1-2004$

\title{
Random Walks on the Torus with Several Generators
}

Timothy Prescott '02

Harvey Mudd College

Francis E. Su

Harvey Mudd College

\section{Recommended Citation}

Timothy Prescott and Francis Edward Su. Random walks on the torus with several generators. Random Structures and Algorithms, 25(3):336-345, 2004.

This Article - preprint is brought to you for free and open access by the HMC Faculty Scholarship at Scholarship @ Claremont. It has been accepted for inclusion in All HMC Faculty Publications and Research by an authorized administrator of Scholarship @ Claremont. For more information, please contact scholarship@cuc.claremont.edu. 


\title{
RANDOM WALKS ON THE TORUS WITH SEVERAL GENERATORS
}

\author{
TIMOTHY PRESCOTT* AND FRANCIS EDWARD SU**
}

\begin{abstract}
Given $n$ vectors $\left\{\vec{\alpha}_{i}\right\}_{i=1}^{n} \in[0,1)^{d}$, consider a random walk on the $d$ dimensional torus $\mathbb{T}^{d}=\mathbb{R}^{d} / \mathbb{Z}^{d}$ generated by these vectors by successive addition and subtraction. For certain sets of vectors, this walk converges to Haar (uniform) measure on the torus. We show that the discrepancy distance $D\left(Q^{* k}\right)$ between the $k$-th step distribution of the walk and Haar measure is bounded below by $D\left(Q^{* k}\right) \geq C_{1} k^{-n / 2}$, where $C_{1}=C(n, d)$ is a constant. If the vectors are badly approximated by rationals (in a sense we will define) then $D\left(Q^{* k}\right) \leq C_{2} k^{-n / 2 d}$ for $C_{2}=C\left(n, d, \vec{\alpha}_{j}\right)$ a constant.
\end{abstract}

Let $\mathbb{T}^{d}=\mathbb{R}^{d} / \mathbb{Z}^{d}$ denote the $d$-dimensional torus. As a quotient group of $\mathbb{R}^{d}$ it is an additive group, so the group elements may be viewed as elements of $[0,1)^{d}$, with the group operation defined as coordinate-wise addition mod 1.

Let $\vec{\alpha}_{1}, \vec{\alpha}_{2}, \ldots, \vec{\alpha}_{n}$ be vectors in $\mathbb{T}^{d}=[0,1)^{d}$, and consider the random walk on the $d$-dimensional torus $\mathbb{T}^{d}$ that proceeds as follows. Start at $\overrightarrow{0}$. At each step, choose one the vectors $\vec{\alpha}_{i}$ with probability $1 / n$ and add or subtract that vector (with probability $1 / 2$ ) to the current position to get to the next position in the walk.

As a random walk on a group, the $k$-th step distribution of the walk converges to a limiting distribution [6], and in many cases this will be Haar measure, the unique translation-invariant measure on the group. For the torus $\mathbb{T}^{d}$, Haar measure may be thought of as the uniform distribution on the "flat" cube $[0,1)^{d}$, since addition corresponds to translation on $\mathbb{R}^{d} / \mathbb{Z}^{d}$. We shall prove bounds for how quickly this random walk approaches Haar measure on the torus.

\footnotetext{
*Department of Mathematics, University of California, Los Angeles, CA 90095, tmpresco@math.ucla.edu.

** Research partially supported by NSF Grant DMS-0301129. Department of Mathematics, Harvey Mudd College, Claremont, CA 91711, su@math.hmc.edu (corresponding author).
} 
We first note that for certain sets of vectors, this walk may not converge to Haar measure. For instance, if all the entries of each $\vec{\alpha}_{i}$ are rational, then the random walk will not converge to Haar measure, but will converge to a limiting distribution supported on a discrete subgroup of $\mathbb{T}^{d}$. As another example, if there is only one generator $\vec{\alpha}_{1}=(x, x, \ldots, x)$ for some irrational $x$, then the walk will be supported on a circle along the "diagonal" of the torus. (However, a single vector can generate a walk that does converge to Haar measure, provided it is chosen well.)

Let $Q$ denote the generating measure for this random walk, i.e., if $S=\cup_{i=1}^{n}\left\{+\vec{\alpha}_{i},-\vec{\alpha}_{i}\right\}$ is the set of generators of the random walk, then for a set $B \subseteq \mathbb{T}^{d}$, let $Q(B)=$ $|B \cap S| /|S|$ where $|\cdot|$ denotes the size of a finite set. The $k$-th step probability distribution is then given by the $k$-th convolution power of $Q$, which we denote by $Q^{* k}$. Let $U$ denote Haar measure.

As a measure of distance between the probability distributions $Q^{* k}$ and $U$, we will use the discrepancy metric, which is defined to be the supremum of the difference of two probability measures over all "boxes" in $\mathbb{T}^{d}=\mathbb{R}^{d} / \mathbb{Z}^{d}$ with sides parallel to the axes in $\mathbb{R}^{d}$, i.e., of the form $\left[a_{1}, b_{1}\right) \times\left[a_{2}, b_{2}\right) \times \ldots \times\left[a_{d}, b_{d}\right)$. Let $D\left(Q^{* k}\right)$ denote the discrepancy of $Q^{* k}$ from Haar measure $U$ :

$$
D\left(Q^{* k}\right):=\sup _{b o x B \subseteq \mathbb{T}^{d}}\left|Q^{* k}(B)-U(B)\right|
$$

The discrepancy metric has been used by number theorists to study the uniform distribution of sequences $\bmod 1$, e.g., see [2, 7]. Diaconis [1] suggested its use for the study of rates of convergence for random walks on groups. It admits Fourier bounds 4] and has many other nice properties and connections with other probability metrics [3].

Although the total variation metric is more commonly used to study the convergence of random walks, we do not use it here because this random walk does not converge in total variation (in fact, the total variation distance between $Q^{* k}$ and $U$ is always 1 , since at any step $Q^{* k}$ is supported on a finite set). The possibility of using Fourier analysis to bound the discrepancy distance makes it a more desirable 
choice than other common metrics on probabilities, such as the Prohorov metric, and has allowed many recent results for the study of discrete random walks on continuous state spaces (e.g., [12, 14]). Most of the literature for rates of convergence of random walks have been limited to walks on finite groups or state spaces, and those that have focused on infinite compact groups (e.g., 8], 9], 13]) have studied walks generated by continuous measures. By contrast, the walk we study is generated by a discrete set of generators on an infinite group.

We prove:

Theorem 1. Let $Q$ denote the generating measure of the the random walk on the $d$-torus generated by $n$ vectors $\vec{\alpha}_{1}, \ldots, \vec{\alpha}_{n}$. Then the $k$-th step probability distribution $Q^{* k}$ satisfies:

$$
D\left(Q^{* k}\right) \geq \frac{1}{\pi^{d} 5^{n+1} d^{n / 2}} k^{-n / 2}
$$

This result holds for any set of vector generators. On the other hand, for certain sets of badly approximable generators (to be defined later), we can establish the following upper bound.

Theorem 2. Let $A_{n \times d}$ be a badly approximable matrix, with rows $\vec{\alpha}_{1}, \ldots, \vec{\alpha}_{n}$, and approximation constant $C_{A}$. If $Q$ is the generating measure of the random walk on the d-torus generated by the $\vec{\alpha}_{i}$, then the $k$-th step probability distribution $Q^{* k}$ satisfies:

$$
D\left(Q^{* k}\right) \leq\left(\frac{3}{2}\right)^{d} 20\left(\frac{n}{C_{A} \sqrt{2}}\right)^{n / d} k^{-n / 2 d} .
$$

We note that the case $d=1$ corresponds to a random walk on the circle, which has been studied for a single generator [12] and for several generators [4].

\section{Lower Bound}

The following notation will be used throughout this paper:

$\|x\|$ : the Euclidean $\left(L^{2}\right)$ norm of a vector $x$ $\|x\|_{\infty}$ : the supremum norm of a vector $x$

$\{x\}$ : the Euclidean $\left(L^{2}\right)$ distance from $x$ to the nearest integral point 
$\{x\}_{\infty}$ : the supremum distance from $x$ to the nearest integral point

To establish a lower bound for the discrepancy, we use a lemma due to Dirichlet:

Lemma 3 (Dirichlet 1842). Given any real $n \times d$ matrix $A$ and $q \geq 1$, there is some $\mathbf{h} \in \mathbb{Z}^{d}$ such that $0<\|\mathbf{h}\|_{\infty} \leq q^{n / d}$ and $\{A \mathbf{h}\}_{\infty}<1 / q$.

A simple proof using a pigeonhole argument may be found in [11]. We now prove Theorem [1]

Proof. Su 13] has shown that for any probability distribution $P$ on $\mathbb{T}^{d}$ :

$$
D(P) \geq \sup _{\mathbf{r} \in(0, .5]^{d}}\left[\sum_{\mathbf{0} \neq \mathbf{h} \in \mathbb{Z}^{d}}|\hat{P}(\mathbf{h})|^{2} \prod_{i=1}^{d}\left\{\begin{array}{ll}
\frac{\sin ^{2}\left(2 \pi h_{i} r_{i}\right)}{\pi^{2} h_{i}^{2}} & \text { if } h_{i} \neq 0 \\
4 r_{i}^{2} & \text { if } h_{i}=0
\end{array}\right\}\right]^{1 / 2}
$$

where $\hat{P}(\mathbf{h})$ is the Fourier transform of $P$, i.e., $\hat{P}(\mathbf{h})=\int_{\mathbb{T}^{d}} e^{2 \pi i \mathbf{h} \cdot \mathbf{x}} Q(d \mathbf{x})$. We will use this formula to bound $D\left(Q^{* k}\right)$ where $Q$ is the generating measure of our random walk. Note that:

$$
\begin{aligned}
\hat{Q}(\mathbf{h}) & =\sum_{j=1}^{n} \frac{1}{2 n}\left(e^{2 \pi i \mathbf{h} \cdot \vec{\alpha}_{j}}+e^{-2 \pi i \mathbf{h} \cdot \vec{\alpha}_{j}}\right) \\
& =\frac{1}{n} \sum_{j=1}^{n} \cos \left(2 \pi \mathbf{h} \cdot \vec{\alpha}_{j}\right) .
\end{aligned}
$$

Since $\cos (2 \pi x)=\cos (2 \pi\{x\}) \geq 1-2 \pi^{2}\{x\}^{2}$, we have

$$
\begin{aligned}
\hat{Q}(\mathbf{h}) & \geq \frac{1}{n} \sum_{j=1}^{n} 1-2 \pi^{2}\left\{\vec{\alpha}_{j} \cdot \mathbf{h}\right\}^{2} \\
& \geq 1-\frac{2 \pi^{2}}{n} \sum_{j=1}^{n}\left\{\vec{\alpha}_{j} \cdot \mathbf{h}\right\}^{2} \\
& \geq 1-\frac{2 \pi^{2}}{n}\{A \mathbf{h}\}^{2}
\end{aligned}
$$

where $A \equiv\left(\vec{\alpha}_{1} \vec{\alpha}_{2} \cdots \vec{\alpha}_{n}\right)^{\top}$ is the $n \times d$ matrix whose rows are the $\alpha_{j}$ 's. Also, noting that $\widehat{Q^{* k}}(\mathbf{h})=\hat{Q}^{k}(\mathbf{h})$ and that $(1-x)^{k} \geq 1-k x$ for $k \geq 1$ and $x \leq 1$, we have that

$$
\widehat{Q^{* k}}(\mathbf{h}) \geq 1-\frac{2 \pi^{2} k}{n}\{A \mathbf{h}\}^{2}
$$


as long as $2 \pi^{2} k\{A \mathbf{h}\}^{2} / n<1$. This is ensured by setting $Z_{1}=2 \pi^{2} / 25<1$ and letting $q=\left(2 \pi^{2} k d / Z_{1}\right)^{1 / 2}$. Then Lemma 3 implies that there exists $\mathbf{h} \in \mathbb{Z}^{d}$ such that $0<\|\mathbf{h}\|_{\infty} \leq q^{n / d}$ and $\{A \mathbf{h}\}_{\infty}<1 / q$. This yields $2 \pi^{2} k\{A \mathbf{h}\}^{2} / n \leq 2 \pi^{2} k d\{A \mathbf{h}\}_{\infty}^{2}<$ $2 \pi^{2} k d / q^{2}=Z_{1}<1$, as desired. (Note that $|\hat{Q}(\mathbf{h})|^{k} \geq 1-Z_{1}$.) By evaluating inequality (11) at this $\mathbf{h}$ we find

$$
D\left(Q^{* k}\right) \geq \sup _{\mathbf{r} \in(0, .5]^{d}}\left[\left(1-Z_{1}\right) \prod_{i=1}^{d}\left\{\begin{array}{ll}
\frac{\sin \left(2 \pi h_{i} r_{i}\right)}{\pi h_{i}} & \text { if } h_{i} \neq 0 \\
2 r_{i} & \text { if } h_{i}=0
\end{array}\right\}\right]
$$

Then, if we let $r_{i}=1 / 4 h_{i}<1 / 2$ if $h_{i} \neq 0$ and $r_{i}=1 / 2 \pi$ if $h_{i}=0$ and define $R(\mathbf{h})=\prod_{i=1}^{n} \max \left\{1,\left|h_{i}\right|\right\}$ to relate the size of $\mathbf{h}$, we find that

$$
\begin{aligned}
D\left(Q^{* k}\right) & \geq\left(1-Z_{1}\right) \prod_{i=1}^{d}\left\{\begin{array}{ll}
\frac{1}{\pi h_{i}} & \text { if } h_{i} \neq 0 \\
\frac{1}{\pi} & \text { if } h_{i}=0
\end{array}\right\} \\
& \geq \frac{1-Z_{1}}{\pi^{d} R(\mathbf{h})} \\
& \geq \frac{1-Z_{1}}{\pi^{d}\|\mathbf{h}\|_{\infty}^{d}} \\
& \geq \frac{1-Z_{1}}{\pi^{d} q^{n}} \\
& \geq \frac{1-Z_{1}}{\pi^{d}\left(2 \pi^{2} k d / Z_{1}\right)^{n / 2}} \\
& \geq \frac{1-Z_{1}}{\pi^{d} 5^{n} d^{n / 2}} k^{-n / 2} \\
& \geq \frac{1}{\pi^{d} 5^{n+1} d^{n / 2}} k^{-n / 2} .
\end{aligned}
$$

\section{UPPER BOUND}

We now seek an upper bound on the discrepancy of the random walk when our generators arise as rows of a badly approximable matrix.

Definition 4. We say an $n \times d$ matrix $A$ is badly approximable if there exists a constant $C_{A}$ such that $\{A \mathbf{h}\}_{\infty}>C_{A} /\|\mathbf{h}\|_{\infty}^{d / n}$ for all non-zero $\mathbf{h} \in \mathbb{Z}^{d}$. We call $C_{A}$ the approximation constant of $A$. 
Note that Lemma 3 implies that for any matrix $A$ (not just badly approximable ones), $\{A \mathbf{h}\}_{\infty}<1 /\|\mathbf{h}\|_{\infty}^{d / n}$ for infinitely many $\mathbf{h} \in \mathbb{Z}^{d}$. Thus we say $A$ is badly approximable if the reverse inequality holds (up to a constant $C_{A}$ ) for all $\mathbf{h} \in \mathbb{Z}^{d}$. This definition closely follows Schmidt [10, who defines badly approximable linear forms; this corresponds to our definition by noting $A \mathbf{h}$ is a linear form in the variables $h_{i}$.

As a subset of $\mathbb{R}^{n d}$, the set of badly approximable matrices has Lebesgue measure zero [5] although their Hausdorff dimension is nd and there are uncountably many of them [10].

We now prove Theorem 2 .

Proof. It is known [2] from Erdős, Turàn, and Koksma that for all positive integers $M$

$$
D\left(Q^{* k}\right) \leq\left(\frac{3}{2}\right)^{d}\left(\frac{2}{M+1}+\sum_{\substack{\mathbf{h} \in \mathbb{Z}^{d} \\ 0<\|\mathbf{h}\|_{\infty} \leq M}} \frac{\left|\hat{Q}^{k}(\mathbf{h})\right|}{R(\mathbf{h})}\right) .
$$

Since $|\cos (2 \pi x)| \leq 1-4\{2 x\}^{2}$ for all $x \in \mathbb{R}$, it follows that

$$
\begin{aligned}
|\hat{Q}(\mathbf{h})| & =\frac{1}{n} \sum_{j=1}^{n}\left|\cos \left(2 \pi \mathbf{h} \cdot \vec{\alpha}_{j}\right)\right| \\
& \leq \frac{1}{n} \sum_{j=1}^{n} 1-4\left\{2 \mathbf{h} \cdot \vec{\alpha}_{j}\right\}^{2} \\
& \leq 1-\frac{4}{n} \sum_{j=1}^{n}\left\{2 \vec{\alpha}_{j} \cdot \mathbf{h}\right\}^{2} \\
& \leq 1-\frac{4}{n}\{2 A \mathbf{h}\}^{2} \\
& \leq \exp \left(-\frac{4}{n}\{2 A \mathbf{h}\}^{2}\right) .
\end{aligned}
$$

In light of inequality (2), we need to estimate a sum of the form

$$
\sum_{0<\|\mathbf{h}\|_{\infty} \leq M} \frac{\left|\hat{Q}^{k}(\mathbf{h})\right|}{R(\mathbf{h})} \leq \sum_{\substack{\mathbf{h} \in \mathbb{Z}^{d} \\ 0<\|\mathbf{h}\|_{\infty} \leq M}} \frac{\exp \left(-\frac{4 k}{n}\{2 A \mathbf{h}\}^{2}\right)}{R(\mathbf{h})}=: S .
$$


Since $M$ may be chosen freely, choose an integer $M$ such that

$$
M \leq \frac{1}{8}\left(\frac{2 k\left(C_{A}\right)^{2}}{n^{2}}\right)^{n / 2 d}<M+1 .
$$

Here $C_{A}$ is an approximation constant for the badly approximable $A$ and $k$ is the number of steps in the walk. We can show:

Lemma 5. With $S$ and $M$ defined as above, $S \leq \frac{0.5}{M+1}$.

Before proving this lemma, we show how the theorem follows. From inequality (21), we find

$$
D\left(Q^{* k}\right) \leq\left(\frac{3}{2}\right)^{d}\left(\frac{2}{M+1}+S\right) \leq\left(\frac{3}{2}\right)^{d}\left(\frac{2}{M+1}+\frac{0.5}{M+1}\right)=\left(\frac{3}{2}\right)^{d} \frac{2.5}{M+1} .
$$

From the inequality for $M+1$ in (3), we have

$$
D\left(Q^{* k}\right) \leq\left(\frac{3}{2}\right)^{d} 20\left(\frac{n^{2}}{2 k\left(C_{A}\right)^{2}}\right)^{n / 2 d}=\left(\frac{3}{2}\right)^{d} 20\left(\frac{n}{C_{A} \sqrt{2}}\right)^{n / d} k^{-n / 2 d},
$$

which concludes the proof of Theorem 2

All that remains is to prove Lemma 5

Proof of Lemma 5. We shall bound $S$ in three stages: (1) first, we group the terms of $S$ into "cohorts" based on the size of $\|\mathbf{h}\|_{\infty},(2)$ we note that the points $2 A \mathbf{h}$ are bounded away from each other in $\mathbb{T}^{d}$ and therefore can bound the terms within each cohort based on the size of $\{2 A \mathbf{h}\}_{\infty}$, and (3) estimating the resulting expression.

(1) Grouping the terms of $S$ by the size of $\|\mathbf{h}\|_{\infty}$. Choose an integer $J$ such that

$$
2^{J-1} \leq M \leq 2^{J}-1
$$


The sum in $S$ may be grouped into $J$ cohorts of integers $\|\mathbf{h}\|_{\infty} \in H_{j}:=\left[2^{j-1}, 2^{j}-1\right]$ for $j=\{1, \ldots, J\}$. Therefore,

$$
\begin{aligned}
S & \leq \sum_{j=1}^{J} \sum_{\|\mathbf{h}\|_{\infty} \in H_{j}} \frac{\exp \left(-\frac{4 k}{n}\{2 A \mathbf{h}\}^{2}\right)}{R(\mathbf{h})} \\
& \leq \sum_{j=1}^{J} \sum_{\|\mathbf{h}\|_{\infty} \in H_{j}} \frac{\exp \left(-\frac{4 k}{n}\{2 A \mathbf{h}\}^{2}\right)}{2^{j-1}} \\
& \leq \sum_{j=1}^{J} \frac{1}{2^{j-1}} \sum_{\|\mathbf{h}\|_{\infty} \in H_{j}} \exp \left(-\frac{4 k}{n}\{2 A \mathbf{h}\}^{2}\right) \\
& \leq \sum_{j=1}^{J} \frac{1}{2^{j-1}} \sum_{\|\mathbf{h}\|_{\infty} \in H_{j}} \exp \left(-\frac{4 k}{n}\{2 A \mathbf{h}\}_{\infty}^{2}\right) .
\end{aligned}
$$

(2) Bounding the terms within each cohort. Within each cohort $\left[2^{j-1}, 2^{j}-1\right]$, since $\mathbf{h}$ is a non-zero integral vector, the use of Definition 4 yields $\{2 A \mathbf{h}\}_{\infty}>C_{A} /\|2 \mathbf{h}\|_{\infty}^{d / n}$ where $C_{A}$ is the approximation constant of the matrix $A$. Therefore, each $2 A \mathbf{h}$ is bounded away from any integral point by $C_{A} /\|2 \mathbf{h}\|_{\infty}^{d / n}$. In fact, they are also bounded away from each other, since if $\left\|\mathbf{h}_{1}\right\|_{\infty},\left\|\mathbf{h}_{2}\right\|_{\infty} \in\left[2^{j-1}, 2^{j}-1\right]$ and $\mathbf{h}_{1} \neq \mathbf{h}_{2}$, then $\left\|\mathbf{h}_{1}-\mathbf{h}_{2}\right\|_{\infty} \leq 2^{j+1}$ and

$$
\left\{2 A\left(\mathbf{h}_{1}-\mathbf{h}_{2}\right)\right\}_{\infty}>\frac{C_{A}}{2^{d / n}\left\|\mathbf{h}_{1}-\mathbf{h}_{2}\right\|_{\infty}^{d / n}} \geq \frac{C_{A}}{2^{d / n}\left(2^{j+1}\right)^{d / n}}=\frac{C_{A}}{2^{(j+2) d / n}} .
$$

Therefore, we divide the unit cube $[0,1]^{n}$ into subcubes of side-length $C_{A} /\left(2^{d(j+2) / n}\right)$ and distribute the points $\{2 A \mathbf{h}\}$ throughout them. In the worst case, all of the points are distributed near the corners of the cube and occupy adjacent subcubes. Therefore,

$$
\begin{aligned}
S & \leq \sum_{j=1}^{J} \frac{2^{n}}{2^{j-1}} \sum_{i=1}^{\left|H_{j}\right|^{1 / n}}(i+1)^{n-1} \exp \left(-\frac{4 k}{n}\left(\frac{i C_{A}}{2^{(j+2) d / n}}\right)^{2}\right) \\
& \leq \sum_{j=1}^{J} 2^{n+1-j} \sum_{i=1}^{\infty}(i+1)^{n-1} \exp \left(-\frac{4 k i^{2}\left(C_{A}\right)^{2}}{n 2^{2(j+2) d / n}}\right)
\end{aligned}
$$


(3) Estimating the resulting expression. Since $M \geq 2^{J-1}$ and

$$
k \geq \frac{n^{2} 2^{6 d / n} M^{2 d / n}}{2\left(C_{A}\right)^{2}} \geq \frac{n^{2} 2^{6 d / n}\left(2^{J-1}\right)^{2 d / n}}{2\left(C_{A}\right)^{2}}=\frac{n^{2} 2^{2(J+2) d / n}}{2\left(C_{A}\right)^{2}},
$$

we can say that:

$$
S \leq \sum_{j=1}^{J} 2^{n+1-j} \sum_{i=1}^{\infty}(i+1)^{n-1} \exp \left(-2 i^{2} n\left(2^{J-j}\right)^{2 d / n}\right) .
$$

Since $j \leq J, i \geq 1$ and $n \geq 1$, the log derivative with respect to $i$ of the inner sum can be bounded:

$$
\frac{n-1}{i+1}-4 i n\left(2^{J-j}\right)^{2 d / n} \leq \frac{n-1}{i+1}-4 i n \leq-4 i \leq-4 .
$$

Therefore, the expression in the inner sum decreases geometrically by at least the ratio $e^{-4}$, and the inner sum can be bounded by the first term (at $i=1$ ) times the constant $1 /\left(1-e^{-4}\right)$. Therefore,

$$
\begin{aligned}
S & \leq \sum_{j=1}^{J} \frac{2^{n+1-j}}{1-e^{-4}} 2^{n-1} \exp \left(-2 n 2^{2(J-j) d / n}\right) \\
& \leq \frac{2^{2 n}}{1-e^{-4}} \sum_{j=1}^{J} 2^{-j} \exp \left(-2 n 2^{2(J-j) d / n}\right) .
\end{aligned}
$$

This sum may be bounded by noting that the largest term occurs when $j=$ $J$. For $j \leq J$, the $\log$ derivative of the terms with respect to $j$ is $\ln 2(-1+$ $\left.d 2^{2+2(J-j) d / n}\right) \geq \ln 2\left(-1+2^{2}\right)=\ln 8$. Therefore, the sum decreases geometrically with ratio at least $1 / 8$ as $j \leq J$ decreases, so the sum is bounded by seven-eighths the final term at $j=J$. Also, recalling that $M \leq 2^{J}-1$,

$$
\begin{aligned}
S & \leq \frac{2^{2 n}}{1-e^{-4}} \frac{7}{8} 2^{-J} \exp \left(-2 n 2^{2(J-J) d / n}\right)=\frac{7}{8\left(1-e^{-4}\right)}\left(\frac{2}{e}\right)^{2 n} \frac{1}{M+1} \\
& \leq \frac{28}{8\left(e^{2}-e^{-2}\right)} \frac{1}{M+1} \leq \frac{0.5}{M+1}
\end{aligned}
$$

as was to be shown.

So, for badly approximable matrices $A$, we have the following discrepancy bounds on the associated random walk:

$$
\frac{C(n, d)}{k^{n / 2}} \leq D\left(Q^{* k}\right) \leq \frac{C(n, d, A)}{k^{n / 2 d}} .
$$


In general, these bounds do not match unless $d=1$. In that case, we recover the same order of convergence as in 4 .

We conjecture that in the lower bound of Theorem 1 (which applies to any random walk on the torus generated by a finite set of vectors) the $k^{n / 2}$ may be improved to $k^{n / 2 d}$ (for all matrices, not just badly approximable ones). This would yield matching upper and lower bounds for random walks on the $d$-torus generated by the rows of a badly approximable matrix. This would confirm that such walks converge the fastest among all finitely-generated random walks on the $d$-torus, a fact that has already been shown in [4] for dimension $d=1$. Developing an approximation lemma similar to Dirichlet's Lemma (Lemma 3) that bounds $R(\mathbf{h})$ instead of $\|\mathbf{h}\|_{\infty}$ may help in this regard.

\section{REFERENCES}

[1] Diaconis, P. Group Representations in Probability and Statistics, Institute of Mathematical Statistics Lecture Notes, Vol. 11, Hayward, CA, 1988.

[2] Drmota, M, and Tichy, R.F. Sequences, Discrepancies and Applications, Lecture Notes in Math. 1651, 66-71, Springer-Verlag, 1997.

[3] Gibbs, A. and Su, F.E. On choosing and bounding probability metrics. Internat. Statist. Rev. (2002), vol. 70, no. 3, 419-435.

[4] Hensley, D, and Su, F.E. Random walks with badly approximable numbers, Unusual Applications of Number Theory, 95-102, DIMACS Ser. Discrete Math. Theoret. Comput. Sci. 64, Amer. Math. Soc., 2004.

[5] Khintchine, A. Zur metrischen Theorie der diophantischen Approximationen, Math. Z. 24, 706-714, 1926.

[6] Kloss, B.M. Limiting distributions on bicompact topological groups. Theory Probab. Appl. 4(1959), 237-270.

[7] Kuipers, L. and Niederreiter, H. Uniform Distribution of Sequences, Wiley, New York, 1974.

[8] Porod, U. The cut-off phenomenon for random reflections, Ann. Probab. 24(1996), 74-96.

[9] Rosenthal, J.S. Random rotations: characters and random walks on $S O(n)$, Ann. Probab. 22(1994), 398-423.

[10] Schmidt, W.M. Badly approximable systems of linear forms, Journal of Number Theory 1 , $139-154,1969$.

[11] Schmidt, W.M. Diophantine Approximation, Lecture Notes in Math. 785, Springer-Verlag, 1980.

[12] Su, F.E. Convergence of random walks on the circle generated by an irrational rotation, Trans. Amer. Math. Soc 350 (1998), 3717-3741.

[13] Su, F.E. A LeVeque type lower bound for discrepancy, Monte Carlo Methods and QuasiMonte Carlo Methods 1998, 448-458, Springer-Verlag, 2000.

[14] Su, F.E. Discrepancy convergence for the drunkard's walk on the sphere, Electron. J. Probab. 6(2001), no. 2, 1-20. 